\title{
Ensemble Multi-objective Biogeography-Based Optimization with Application to Automated Warehouse Scheduling
}

\author{
Haiping $\mathrm{Ma}^{1,2^{*}}$, Shufei $\mathrm{Su}^{3}$, Dan Simon ${ }^{4}$, and Minrui Fei ${ }^{2}$ \\ ${ }^{1}$ Department of Electrical Engineering, Shaoxing University, Shaoxing, Zhejiang, China \\ ${ }^{2}$ Shanghai Key Laboratory of Power Station Automation Technology, School of Mechatronic \\ Engineering and Automation, Shanghai University, Shanghai, China \\ ${ }^{3}$ School of Communication and Information Engineering, Shanghai University, Shanghai, China \\ ${ }^{4}$ Department of Electrical and Computer Engineering, Cleveland State University, \\ Cleveland, Ohio, USA
}

\begin{abstract}
This paper proposes an ensemble multi-objective biogeography-based optimization (EMBBO) algorithm, which is inspired by ensemble learning, to solve the automated warehouse scheduling problem. First, a real-world automated warehouse scheduling problem is formulated as a constrained multi-objective optimization problem. Then EMBBO is formulated as a combination of several multi-objective biogeography-based optimization (MBBO) algorithms, including vector evaluated biogeography-based optimization (VEBBO), non-dominated sorting biogeography-based optimization (NSBBO), and niched Pareto biogeography-based optimization (NPBBO).
\end{abstract} Performance is tested on a set of 10 unconstrained multi-objective benchmark functions and 10 constrained multi-objective benchmark functions from the 2009 Congress on Evolutionary Computation (CEC), and compared with single constituent $\mathrm{MBBO}$ and $\mathrm{CEC}$ competition algorithms. Results show that EMBBO is better than its constituent algorithms, and among the best CEC competition algorithms, for the benchmark functions studied in this paper. Finally, EMBBO is 
successfully applied to the automated warehouse scheduling problem, and the results show that EMBBO is a competitive algorithm for automated warehouse scheduling.

Keywords: automated warehousing; travel time analysis; multi-objective optimization; simulation; performance analysis

\section{Introduction}

Warehousing is an important part of production supply chain management, and serves as the backbone in many manufacturing enterprises. Warehousing keeps stocks of products until they are ready to be delivered to the market place. A delay in product delivery may lead to the failure of production supply chains. Efficient warehouse management contributes to the timely delivery of the product (Choi et al., 2013; Yeung et al., 2010, 2011). Modern warehouses are equipped with storage and retrieval (S/R) machines to pick up products from an input/output (I/O) location and store them at specific locations, and then to retrieve outgoing products from other storage locations and deliver them to the $\mathrm{I} / \mathrm{O}$ location. Although $\mathrm{S} / \mathrm{R}$ machines enhance warehouse management, scheduling is a challenging and vital task (Lerher, et al., 2015b; Wang and Fang, 2011). The time and cost of product allocation and delivery are important variables to consider during warehouse scheduling.

In the past few decades, warehouse scheduling has been the subject of much research, including energy efficient design (Lerher et al., 2013), travel time models for shuttle-based systems (Lerher et al., 2015a.), travel time models for aisle transfer systems (Lerher et al., 2010), travel time models for double-deep systems (Lerher et al., 2010), and models for mini-load multi-shuttle systems (Lerher et al., 2011). Other automated warehouse schedule research is reported in (Berg, 1999; 
Chan and Kumar, 2009; Roodbergen and Vis, 2009; Yang et al., 2013).

Warehouse scheduling is a typical NP-hard problem, which is one of the most challenging types of combinatorial optimization problems (Gagliardi et al., 2012). Since this problem is so important for production supply chain success, more research needs to be carried out to make automated warehouse scheduling more robust and efficient. Motivated by these considerations, this paper highlights the benefits associated with automated warehouse scheduling and introduces a new evolutionary algorithm to find efficient schedules for warehouse management.

In recent years, ensemble learning has been introduced to enhance the performance of various systems; for example, feature selection, optimization, clustering analysis, etc. Ensemble learning is a hybrid method that uses multiple learning models instead of a single model. This approach is intuitively attractive because a single model may not always be the best to solve a complex problem, but multiple models are likely to yield results that are better than each of the constituent models. Ensemble learning has been successfully applied to time series prediction and classification, and to evolutionary algorithms. It has been used with evolution strategies (ES) (Mallipeddi and Suganthan, 2010b), evolutionary programming (EP) (Mallipeddi and Suganthan, 2010a), harmony search (HS) (Pan et al., 2009), and differential evolution (DE) (Mallipeddi et al., 2011). It has been used to solve constrained optimization (Tasgetiren et al., 2010a), multi-objective optimization (Zhao and Suganthan, 2010), dynamic optimization (Yu and Suganthan, 2009), and the traveling salesman problem (Tasgetiren et al., 2010b).

Biogeography-based optimization (BBO) (Simon, 2008) is an evolutionary global optimization algorithm that was introduced in 2008. It is modeled after the immigration and emigration of species between habitats. The application of these processes to optimization allows information 
sharing between candidate solutions. BBO uses the fitness of each candidate solution to determine its immigration and emigration rate. The emigration rate is proportional to fitness and the immigration rate is inversely proportional to fitness. BBO has demonstrated good performance on various benchmark functions and real-world optimization problems (Ma and Simon, 2011). BBO has also been modified to solve multi-objective optimization problems (MOPs) (Chutima and Wong, 2014; Costa e Silva et al., 2012; Jamuna and Swarup, 2012; Ma et al., 2012).

The aim of this paper is to propose and study an ensemble of multi-objective biogeography-based optimization (MBBO) algorithms, including vector evaluated biogeography-based optimization (VEBBO), non-dominated sorting biogeography-based optimization (NSBBO), and niched Pareto biogeography-based optimization (NPBBO) (Simon, 2013), and apply the new algorithm to the automated warehouse scheduling problem. This paper shows how MBBO algorithms can be integrated to obtain a new algorithm called ensemble multi-objective biogeography-based optimization (EMBBO), and then presents a comparative study on multi-objective benchmark functions and automated warehouse scheduling problems. The methods in this paper could also serve as a template for the extension of any other evolutionary algorithm to multi-objective optimization.

The motivation of proposing EMBBO in this research is two-fold. First, we have observed that ensemble EAs outperform constituent EAs in many applications, as noted above, because of their greater adaptability. Second, we have observed that MBBO has proven itself to be an effective multi-objective optimization algorithm, also noted above. Combining these two observations leads us to propose an ensemble $\mathrm{BBO}$ algorithm, EMBBO, as a high-performing multi-objective optimization algorithm. 
The original contributions of this paper include the following. (a) A new real-world-based automated warehouse scheduling model is formulated as a constrained multi-objective optimization problem. (b) The idea of ensemble learning is applied to MBBO to establish the new EMBBO algorithm. Results show that EMBBO outperforms its constituent MBBO algorithms for most of the unconstrained and constrained multi-objective benchmark functions that we study. (c) EMBBO has a lower computational cost than its constituent algorithms because it simultaneously uses multiple parallel populations to reduce running time in comparison with single $\mathrm{MBBO}$ algorithms which use populations whose number of individuals is the sum of the three constituent algorithms. (d) EMBBO solves the automated warehouse scheduling problem.

The remainder of this paper is organized as follows. Section 2 builds a mathematical model of the automated warehouse scheduling problem. Section 3 reviews the standard BBO and various MBBO algorithms, and then integrates them to realize EMBBO. Section 4 presents performance comparisons on benchmark functions between EMBBO, contintuent MBBO algorithms, and 2009 Congress on Evolutionary Computation (CEC) algorithms, and then applies EMBBO to the automated warehouse scheduling model. Section 5 presents conclusions and suggests directions for future work. The abbreviations, notations, and symbols used in this paper are summarized in the appendix.

\section{Automated Warehouse Scheduling Model}

The layout of the automated warehouse system is shown in Figure 1 (Yang et al., 2013), and is called a multi-aisle automated storage and retrieval system (multi-aisle AS/RS) with a curve-going S/R machine. It includes six components: S/R machine, picking aisles, cross warehouse aisle, storage 
racks (SR), rolling conveyor, and I/O location. As shown in the figure, the S/R machine can go in and out at both ends of every picking aisle, pick up products at the $\mathrm{I} / \mathrm{O}$ location and store them at specific storage units in SR, and then retrieve outgoing products from other storage units and deliver them to the I/O location. The aim of an automated warehouse scheduling system is to improve scheduling efficiency.

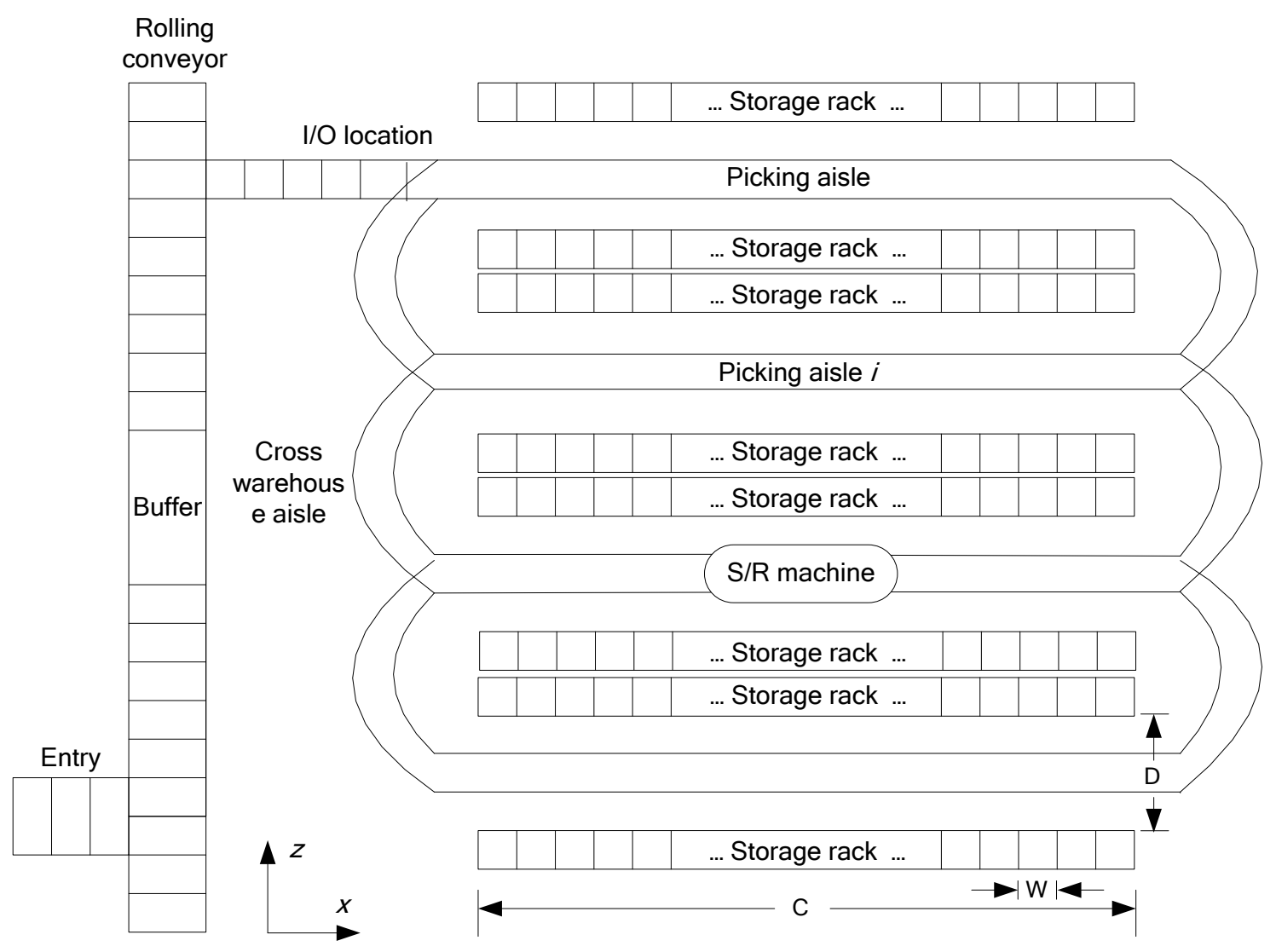

Figure 1 Layout of the warehouse system

In this model, there are many storage units in each SR. There are $m$ storage products, storage units of which are denoted as $\left(p_{u 1}, p_{u 2}, p_{u 3}, \cdots, p_{u m}\right)$. There are $n$ outgoing products, storage units of which are denoted as $\left(p_{o 1}, p_{o 2}, p_{o 3}, \cdots, p_{o n}\right)$. In general, the numbers and units of the storage products are not the same as those of the outgoing products; namely, $m \neq n$ and $p_{u i} \neq p_{o i}$. 
Suppose the S/R machine can hold $N$ products at a time. Then the $\mathrm{S} / \mathrm{R}$ machine picks up $N$ or fewer products from the I/O location, and puts them into storage units. Then it retrieves $N$ or fewer outgoing products from the other storage units, and delivers them to the $\mathrm{I} / \mathrm{O}$ location. For $m$ storage products and $n$ outgoing products, there are $m+n$ storage units, namely, $\left(p_{1}, p_{2}, \cdots, p_{m}, p_{m+1} \cdots, p_{m+n}\right)$, and the S/R machine needs to execute $\max \{\lceil m / N\rceil,\lceil n / N\rceil\}$ tasks to store and transport all products, where $\lceil\varphi\rceil$ denotes the smallest integer greater than or equal to $\varphi$. When each task corresponds to one route, the automated warehouse scheduling problem is translated into an optimization problem of selecting the optimal $\max \{\lceil m / N\rceil,\lceil n / N\rceil\}$ routes from all possible routes to complete the storage/retrieval tasks while satisfying real-world constraints.

In Figure 1, $x$ and $z$ are the two directions in the horizontal plane. The velocities in the $x$ and $z$ directions are called horizontal velocities, and the corresponding horizontal velocities are $v_{x}$ and $v_{z}$, which are equal to each other. $y$ is the vertical direction and the corresponding vertical velocity is $v_{y}$, which is independent from $v_{x}$. The distance between adjacent SRs in the same picking aisle is $D$, and the number of storage units in each SR is $C$. The width, length and height of each storage unit are denoted as $W, L$ and $H$ respectively. The Euclidean coordinates of each storage unit is denoted as $p(x, y, z)$, and the $\mathrm{I} / \mathrm{O}$ location of the $\mathrm{S} / \mathrm{R}$ machine is denoted as $p_{0}(0,0,0)$

When developing the automated warehouse scheduling system, the following assumptions are made:

- The multi-aisle AS/RS is divided into picking aisles with SRs on both sides, so there are double SRs between the picking aisles and a single SR along each warehouse wall.

- $\quad$ There is one S/R machine. 
- The S/R machine is able to move along the cross warehouse aisle by using the curved rails at the end of the picking aisles.

- The S/R machine travels at a constant velocity both in the horizontal and in the vertical directions. Although the inclusion of acceleration and deceleration will affect the scheduling results, it will not affect the optimization approach considered in this paper.

- $\quad$ The S/R machine simultaneously begins the lifting and travelling in the pick aisle. Although the inclusion of non-simultaneous lifting and travelling will affect the scheduling results, it will not affect the optimization approach considered in this paper.

- The input station dwell-point strategy is used. That is, the S/R machine stays always at input location when it is idle. Although the use of other dwell-point strategies will affect the scheduling results, it will not affect the optimization approach considered in this paper.

- The randomized storage assignment policy is used. That is, any storage location within the $\mathrm{S} / \mathrm{R}$ is equally likely to be selected for the storage or retrieval request. Similar to the constant velocity model assumed above, this is a simplified model. Although the use of non-random storage assignment will affect the scheduling results, it will not affect the optimization approach considered in this paper.

- As a first-order approximation, the pickup and set down times, and additional overhead times for manipulating the S/R machine, are ignored.

Definition 1: If the $\mathrm{S} / \mathrm{R}$ machine travels the route $r \in[1, \max (\lceil m / N, n / N\rceil)]$, then $l_{r}=1$; otherwise, $l_{r}=0$. Similarly, if the storage unit $p_{i}$ belongs to route $r$, then $g_{i r}=1$; otherwise, $g_{i r}=0$. 
Definition 2: If the $\mathrm{S} / \mathrm{R}$ machine travels from storage unit $p_{i}\left(x_{i}, y_{i}, z_{i}\right)$ to another storage unit $p_{j}\left(x_{j}, y_{j}, z_{j}\right)$ for $i, j \in[1, m+n]$, then $e_{i j}=1$; otherwise, $e_{i j}=0$. The travel distance $d_{i j}$ and time $t_{i j}$ of the $\mathrm{S} / \mathrm{R}$ machine is denoted as

$$
d_{i j}= \begin{cases}W \times\left|x_{i}-x_{j}\right|+H \times\left|y_{i}-y_{j}\right| & \text { if } z_{i}=z_{j} \\
\min \left(\begin{array}{l}
W \times\left|x_{i}-x_{j}\right|+H \times\left|y_{i}-y_{j}\right|+D \times\left|z_{i}-z_{j}\right|, \\
W \times\left|\left(C-x_{i}\right)-\left(C-x_{j}\right)\right|+H \times\left|y_{i}-y_{j}\right|+D \times\left|z_{i}-z_{j}\right|
\end{array}\right) & \text { if } z_{i} \neq z_{j}\end{cases}
$$

and

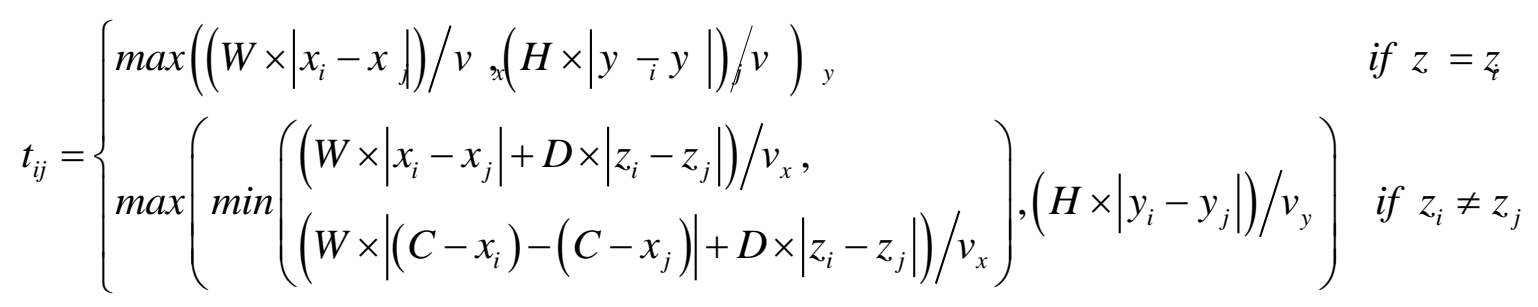

The first expressions of each of the above two equations denote that the two storage units are the same SR because $z_{i}=z_{j}$. The second expressions in each of the equations denote that two storage units are not the same SR because $z_{i} \neq z_{j}$, so we need to first compute the minimum distance that the S/R machine drives between two ends of a picking aisle to compute travel distance and travel time.

Definition 3: The execution time of each task must be less than or equal to the specified scheduling time $T_{r}$. If it is larger than $T_{r}$, it will affect the scheduling quality by an amount equal to the product of the time exceeding $T_{r}$ and the weight coefficient $w_{r}$.

\section{Optimization}

Next, the mathematic model of the automated warehouse scheduling problem is formulated as a multi-objective optimization problem. Suppose the warehouse throughput capacity is $Q$, and the 
number of products in the warehouse is $q$. The automated warehouse scheduling problem has two objectives: the scheduling quality effect should be minimized, and the travel distance should be minimized. The two objectives are defined as follows.

$$
\begin{aligned}
& \min f(e)=\min \left(f_{1}(e), f_{2}(e)\right) \\
& f_{1}(e)=\sum_{r=1}^{\max \{[m / N\rceil,\lceil n / N\rceil\}}\left(\max \left\{0,\left(\sum_{i=1}^{m+n} \sum_{j=1}^{m+n} t_{i j} e_{i j} g_{i r} g_{j r}\right)-T_{r}\right\} \cdot w_{r}\right) \cdot l_{r} \\
& f_{2}(e)=\sum_{r=1}^{\max \{[m / N\rceil,\lceil n / N\rceil\}}\left(\sum_{i=1}^{m+n} \sum_{j=1}^{m+n} d_{i j} e_{i j} g_{i r} g_{j r}\right) \cdot l_{r}
\end{aligned}
$$

where (3) denotes that two objectives are to be minimized, (4) denotes the scheduling quality effect, and (5) denotes the travel distance. Furthermore, the solution must satisfy the following constraints.

Total number of routes

$$
\sum_{r=1}^{\max \{\lceil m / N\rceil,\lceil n / N\rceil\}} l_{r}=\max \{\lceil m / N\rceil,\lceil n / N\rceil\}
$$

Total number of storage and outgoing products

$$
\sum_{r=1}^{\max \{[m / N\rceil,\lceil n / N\rceil\}} \sum_{j=1}^{m+n} g_{j r} l_{r}=m+n
$$

Each storage or outgoing product is handled exactly once

$$
\sum_{r=1}^{\max \{[m / N\rceil,\lceil n / N\rceil\}} g_{j r}=1, \quad \text { for } j \in\{1,2, \cdots, m+n\}
$$

S/R machine load

$$
\sum_{j}^{m+n} g_{j r} \leq 2 N, \text { for } r \in\{1, \cdots, \max \{\lceil m / N\rceil,\lceil n / N\rceil\}\}
$$

Input location

$$
\sum_{r=1}^{\max \{[m / N\rceil,\lceil n / N\rceil\}} e_{0 j} l_{r}=\lceil m / N\rceil, \quad \text { for } j \in\{1,2, \cdots, m+n\}
$$


Output location

$$
\sum_{r=1}^{\max \{\lceil m / N\rceil,\lceil n / N\rceil\}} e_{i 0} l_{r}=\lceil n / N\rceil, \quad \text { for } i \in\{1,2, \cdots, m+n\}
$$

Storage product priorities

$$
p_{u} \in p_{o j} \text { for } r\left(p_{i}, p_{j}, \cdots, p_{u}\right)
$$

Throughput capacity

$$
q+m+n \leq Q
$$

In the above constraints, (6) constrains the number of $S / R$ machine routes to the total number of tasks, (7) constrains the total number of storage and outgoing products, (8) constrains each storage and outgoing product to exactly one route, (9) constrains the total number of storage and outgoing products to no more than twice the S/R machine capacity each route, (10) and (11) constrain the I/O location, where the output location is the same as the input location since the $\mathrm{S} / \mathrm{R}$ machine returns to the input location after each task is completed, (12) constrains storage products to be handled before outgoing products, and (13) constrains the number of products to be no more than the throughput capacity of the warehouse.

Now that a warehouse model and scheduling problem have been presented, the following section develops the optimization algorithm that will be used to solve the warehouse scheduling problem.

\section{Ensemble Multi-objective Biogeography-based Optimization}

This section first reviews BBO (Section 3.1), then reviews several MBBO algorithms, including VEBBO, NSBBO, and NPBBO (Section 3.2), and finally introduces the new EMBBO algorithm (Section 3.3). 


\subsection{Biogeography-based Optimization}

$\mathrm{BBO}$ is an evolutionary algorithm inspired by biogeography to solve general optimization problems. Each candidate solution is comprised of a set of features, which are similar to genes in genetic algorithms (GAs), and which are also called independent variables or decision variables in the optimization literature. Like other evolutionary algorithms, BBO probabilistically shares information between candidate solutions to improve candidate solution fitness. In $\mathrm{BBO}$, each candidate solution immigrates decision variables from other candidate solutions based on its immigration rate, and emigrates decision variables to other candidate solutions based on its emigration rate. In the original BBO paper (Simon, 2008), immigration rates are first used to decide whether or not to immigrate decision variables for a given candidate solution. Then, if immigration is selected for a given candidate solution, emigration rates are used to choose the emigrating candidate solution. Migration can be expressed as

$$
a_{i}(s) \leftarrow a_{j}(s)
$$

where $a_{i}$ denotes the immigrating solution, $a_{j}$ denotes the emigrating solution, and $s$ denotes a decision variable. In $\mathrm{BBO}$, each candidate solution $a$ has its own immigration rate $\lambda$ and emigration rate $\mu$. A good candidate solution has relatively high $\mu$ and low $\lambda$, while the converse is true for a poor candidate solution. The immigration rate and the emigration rate are functions of candidate solution fitness. According to (Simon, 2008), these functions can be calculated as

$$
\begin{aligned}
& \lambda=1-f(a) \\
& \mu=f(a)
\end{aligned}
$$


where $f$ denotes candidate solution fitness, which is normalized to the range $[0,1]$. The probability of immigrating to $a_{i}$ and the probability of emigrating from $a_{j}$ are calculated respectively as

$$
\begin{aligned}
& \text { Prob(immigration to } \left.a_{i}\right)=\lambda_{i} \\
& \text { Prob(emigration from } \left.a_{j}\right)=\frac{\mu_{j}}{\sum_{k=1}^{N_{0}} \mu_{k}}
\end{aligned}
$$

where $N_{0}$ is the population size. After migration, probabilistic mutation occurs for each decision variable of each candidate solution. Mutation of candidate solution $a_{i}$ is implemented as follows.

$$
\begin{aligned}
& \text { For each candidate solution decision variable } s \text { in } a_{i} \\
& \text { If } \operatorname{rand}(0,1)<\theta \\
& \quad a_{i}(s) \leftarrow \operatorname{rand}\left(L_{s}, U_{s}\right)
\end{aligned}
$$$$
\text { End if }
$$

Next $s$

In the above mutation logic, $\operatorname{rand}\left(L_{s}, U_{s}\right)$ is a uniformly distributed random number between $L_{s}$ and $U_{s}, \theta$ is the mutation probability, and $L_{s}$ and $U_{s}$ are the lower and upper search bounds of the sth independent variable. The above logic mutates each independent variable with a probability of $\theta$. If mutation occurs for a given independent variable, the independent variable is replaced with a random number within its search domain. A description of one generation of BBO is given in Algorithm 1. 
Algorithm 1 - One generation of the $\mathrm{BBO}$ algorithm. $a_{i}$ is the $i$ th candidate solution, and $a_{i}(s)$ is the $s$ th decision variable of $a_{i}$.

Biogeography-based optimization (BBO) algorithm

For each $a_{j}$, define emigration rate $\mu_{j}$ proportional to fitness of $a_{j}$, with $\mu_{j} \in[0,1]$

For each $a_{j}$, define immigration rate $\lambda_{j}=1-\mu_{j}$

For each $a_{i}$

For each candidate solution decision variable $s$

Use $\lambda_{i}$ to probabilistically decide whether to immigrate to $a_{i}$ (Eq. 16)

If immigrating then

Use $\{\mu\}$ to probabilistically select the emigrating solution $a_{j}$ (Eq. 16)

$$
a_{i}(s) \leftarrow a_{j}(s)
$$

End if

Next candidate solution decision variable

Probabilistically decide whether to mutate $a_{i}$ (see mutation logic following Eq. 16)

Next candidate solution

In Algorithm 1, the statement "use $\lambda_{i}$ to probabilistically decide whether to immigrate to $a_{i}$ " can be implemented with the following logic.

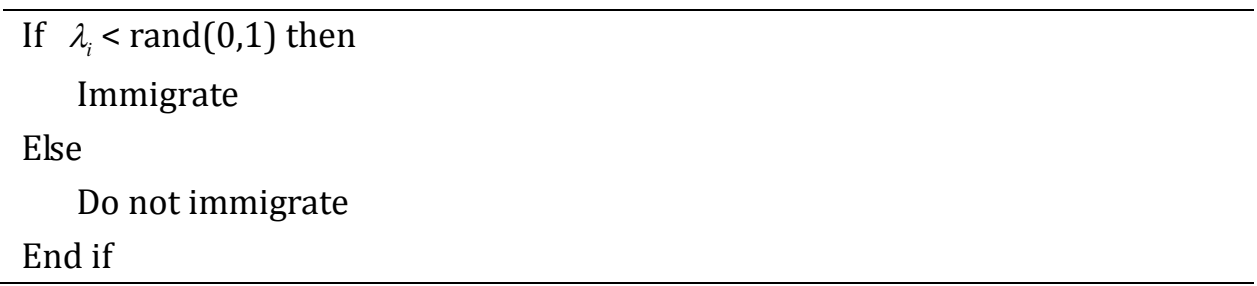

In Algorithm 1, the statement "Use $\{\mu\}$ to probabilistically select the emigrating solution $a_{j}$ " can be implemented with any fitness-based selection method since $\mu$ is proportional to the fitness of $a$. For instance, tournament selection could be used by randomly choosing two or more solutions for a tournament, and then selecting $a_{j}$ as the fittest solution in the tournament. In this paper, as in most other BBO implementations, $\{\mu\}$ is used in a roulette-wheel algorithm so that the 
probability that each individual $a_{j}$ is selected for emigration is proportional to its emigration rate $\mu_{j}$

\subsection{Multi-objective BBO}

This section is based on (Simon, 2013, Section 20.5). First, this section reviews the VEBBO algorithm, which combines BBO with the vector evaluated genetic algorithm (VEGA). Recall that VEGA was one of the original multi-objective evolutionary algorithms (MOEAs), and operates by performing selection on the population using one objective function at a time (Coello et al., 2004; Schaffer, 1985). VEBBO produces a set of subpopulations, one set for each objective function. Then individuals are selected from the subpopulations to obtain parents, which create children by using the BBO migration method. The outline of VEBBO for a $k$-objective optimization problem is shown in Algorithm 2, where MBBO immigration is based on the $k_{i}$ th objective function value of each individual, and $k_{i}$ is a random objective function index at the $i$ th migration trial. Then emigration is based on the $k_{e}$ th objective function value of each individual, where $k_{e}$ is also a random objective function index. 
Algorithm 2 - Outline of VEBBO for solving an $n_{0}$-dimensional optimization problem with $k$ objectives and a population size of $N_{0}$. Each generation, the best individual $a_{b}$ with respect to the $i$ th objective value has rank $\gamma_{b i}=1$, and the worst individual $a_{w}$ has rank $\gamma_{w i}=N_{0}$.

Vector evaluated biogeography-based optimization (VEBBO) algorithm

Randomly initialize a population of candidate solutions $P=\left\{a_{j}\right\}$ for $j \in\left[1, N_{0}\right]$

While termination criterion is not satisfied do

Compute the cost $f_{i}\left(a_{j}\right)$ for each objective $i$ and for each individual $a_{j} \in P$

For each objective $i$ where $i \in[1, k]$ do $\gamma_{j i} \leftarrow$ rank of $a_{j}$ with respect to the $i$ th objective function for $j \in\left[1, N_{0}\right]$

Immigration rates $\lambda_{j i} \leftarrow \gamma_{j i} / \sum_{q=1}^{N_{0}} \gamma_{q i}$ for $j \in\left[1, N_{0}\right], i \in[1, k]$

Emigration rates $\mu_{j i} \leftarrow 1-\lambda_{j i}$ for $j \in\left[1, N_{0}\right], i \in[1, k]$

For each individual $a_{j}$ where $j \in\left[1, N_{0}\right]$ do

For each independent variable $s \in\left[1, n_{0}\right]$ do

$k_{i} \leftarrow \operatorname{rand}(1, k)=$ uniformly distributed integer between 1 and $k$

$\delta \leftarrow \operatorname{rand}(0,1)=$ uniformly distributed real number between 0 and 1

If $\delta<\lambda_{j, k_{i}}$ then

$k_{e} \leftarrow \operatorname{rand}(1, k)=$ uniformly distributed integer between 1 and $k$

Probabilistically select emigrant $a_{e}$, where $\operatorname{Pr}\left(a_{e}=a_{\beta}\right)=\mu_{\beta, k_{e}} / \sum_{q=1}^{N_{0}} \mu_{q, k_{e}}$ for $\beta \in\left[1, N_{0}\right]$ $a_{j}(s) \leftarrow a_{e}(s)$

End if

End for

End for

End for

Probabilistically mutate the population $P$ as described in the standard BBO algorithm

End while

Next, the NSBBO algorithm is reviewed, which combines BBO with the non-dominated sorting genetic algorithm (NSGA). Recall that NSGA was one of the original MOEAs, and assigns the cost of each individual based on its dominance level (Deb et al., 2002; Srinivas and Deb, 1995). First, all individuals are copied to a temporary population $T$. Then we find all non-dominated individuals in $T$; these individuals, which are denoted as the set $B$, are assigned the lowest cost value. Recall that an individual $x$ is dominated by an individual $y$ if $y$ performs at least as good as $x$ in all objectives, 
and performs better than $x$ in at least one objective (Simon, 2013, Chapter 20). An individual is called non-dominated if there are no individuals in the population ( $T$ in this case) that dominate it. Next, $B$ is removed from $T$, and we then find all non-dominated individuals in the reduced set $T$. These individuals are assigned the second-lowest cost value. This process is repeated to obtain a cost for each individual that is based on its level of non-domination. We combine BBO with NSGA by changing the recombination logic in NSGA to BBO migration operations, which results in NSBBO, as shown in Algorithm 3.

Algorithm 3 - Outline of NSBBO for solving an $n_{0}$-dimensional optimization problem with $k$ objectives and a population size of $N_{0}$.

Non-dominated sorting biogeography-based optimization (NSBBO) algorithm

Randomly initialize a population of candidate solutions $P=\left\{a_{j}\right\}$ for $j \in\left[1, N_{0}\right]$

While termination criterion is not satisfied do

Temporary population $T \leftarrow P$

Non-domination level $c \leftarrow 1$

While the temporary population size $|T|>0$ do

$B \leftarrow$ non-dominated individuals in $T$

Cost $f(a) \leftarrow c$ for all $a \in B$

Remove $B$ from $T$

$c \leftarrow c+1$

\section{End while}

Immigration rates $\lambda_{j} \leftarrow f\left(a_{j}\right) / \sum_{q=1}^{N_{0}} f\left(a_{q}\right)$ for $j \in\left[1, N_{0}\right]$

Emigration rates $\mu_{j} \leftarrow 1-\lambda_{j}$ for $j \in\left[1, N_{0}\right]$

For each individual $a_{j}$ where $j \in\left[1, N_{0}\right]$ do

For each independent variable $s \in\left[1, n_{0}\right]$ do

$\delta \leftarrow \operatorname{rand}(0,1)=$ uniformly distributed real number between 0 and 1

If $\delta<\lambda_{j}$ then

Probabilistically select emigrant $a_{e}$, where $\operatorname{Pr}\left(a_{e}=a_{\beta}\right)=\mu_{\beta} / \sum_{q=1}^{N_{0}} \mu_{q}$ for $\beta \in\left[1, N_{0}\right]$ $a_{j}(s) \leftarrow a_{e}(s)$

\section{End if}

\section{End for}

\section{End for}

Probabilistically mutate the population $P$ as described in the standard BBO algorithm 
Finally, the NPBBO algorithm is reviewed, which combines BBO with the niched Pareto genetic algorithm (NPGA). Recall that NPGA was one of the original MOEAs, which is similar to NSGA in its assignment of cost on the basis of domination (Horn, et al., 1994). NPGA is an attempt to reduce the computational effort of NSGA. Two tournament individuals $a_{1}$ and $a_{2}$ are selected randomly from the population, and then a subset $S$ of the population is also randomly selected, which is typically around $10 \%$ of the population. If one of the individuals $a_{1}$ and $a_{2}$ is dominated by any of the individuals in $S$, and the other is not, then the non-dominated individual, denoted as $a_{0}$, wins the tournament and is selected for recombination. If both individuals $a_{1}$ and $a_{2}$ are dominated by at least one individual in $S$, or both individuals are not dominated by any individuals in $S$, then fitness sharing is used to decide the tournament winner; that is, the individual that is in the least crowded region of the objective function space wins the tournament. This selection process can be described as follows.

$$
\begin{aligned}
& b_{i}=\left|y_{0} \in S: y_{0} \succ a_{i}\right| \quad \text { for } i \in[1,2] \\
& c_{i}=\text { Crowding distance of } a_{i} \text { for } i \in[1,2] \\
& a_{0}=\left\{\begin{array}{c}
a_{1} \text { if }\left\{\begin{array}{l}
\left(b_{1}=0\right) \text { and }\left(b_{2}>0\right), \text { or } \\
\left(b_{1}>0\right) \text { and }\left(b_{2}>0\right) \text { and }\left(c_{1}<c_{2}\right), \text { or } \\
\left(b_{1}=0\right) \text { and }\left(b_{2}=0\right) \text { and }\left(c_{1}<c_{2}\right)
\end{array}\right. \\
\text { otherwise }
\end{array}\right.
\end{aligned}
$$

where $y_{0} \succ a_{i}$ denotes that $y_{0}$ dominates $a_{i}$; that is, $y_{0}$ is at least as good as $a_{i}$ for all objective function values, and it is better than $a_{i}$ for at least one objective function value. $b_{i}$ is the number of individuals that dominate $a_{i}, c_{i}$ is the crowding distance of $a_{i}$, and $a_{0}$ is the individual (either $a_{1}$ or $a_{2}$ ) that is finally selected for recombination. The crowding distance $c$ could be computed with a method from (Simon, 2013, pp. 541). For each objective function 
dimension, the closest larger value and the closest smaller value in the population are found as follows.

$$
\begin{aligned}
& f_{j}^{-}(a)=\max _{a^{*}}\left\{f_{j}\left(a^{*}\right) \text { such that } f_{j}\left(a^{*}\right)<f_{j}(a)\right\} \\
& f_{j}^{+}(a)=\min _{a^{*}}\left\{f_{j}\left(a^{*}\right) \text { such that } f_{j}\left(a^{*}\right)>f_{j}(a)\right\}
\end{aligned}
$$

where $f_{j}(a)$ is the objective function value of $a$ with respect to the $j$ th objective. The crowding distance of $a$ is then computed as

$$
c=\sum_{j=1}^{k}\left(f_{j}^{+}(a)-f_{j}^{-}(a)\right)
$$

We combine BBO with NPGA by changing the recombination logic in NPGA to BBO migration operations, which results in NPBBO, as shown as Algorithm 4.

Algorithm 4 - Outline of NPBBO for solving an $n_{0}$-dimensional optimization problem with $k$ objectives and a population size of $N_{0}$.

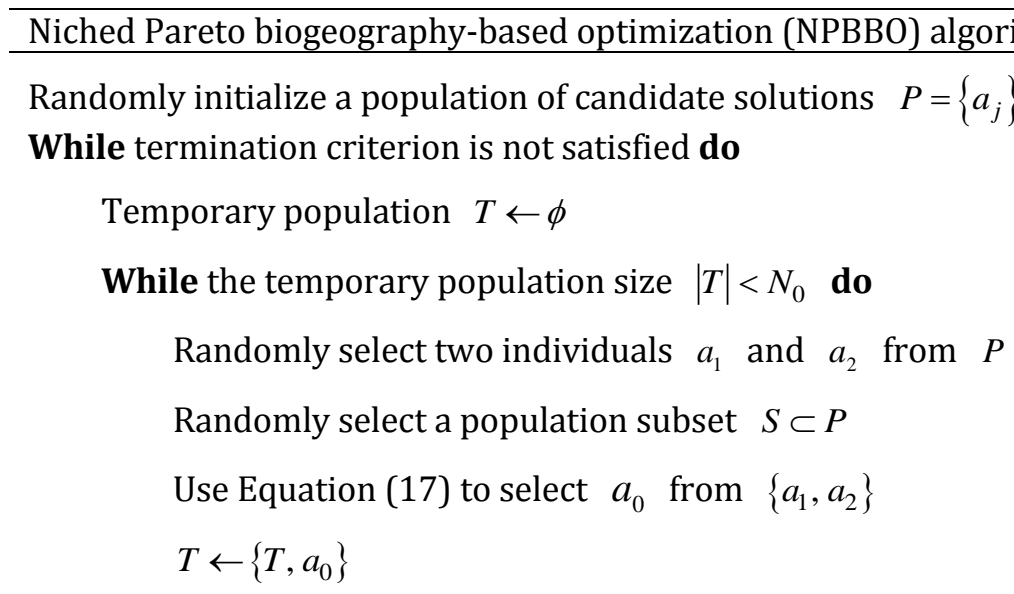

\section{End while}

For each individual $a_{j} \in T$, where $j \in\left[1, N_{0}\right]$ do

For each independent variable $s \in\left[1, n_{0}\right]$ do

$$
\begin{aligned}
& \delta \leftarrow \operatorname{rand}(0,1)=\text { uniformly distributed real number between } 0 \text { and } 1 \\
& \text { If } \delta<1 / N_{0} \text { then } \\
& \quad \text { Probabilistically select emigrant } a_{e} \text {, where } \operatorname{Pr}\left(a_{e}=x_{\beta}\right)=1 / N_{0} \text { for } \beta \in\left[1, N_{0}\right] \\
& \quad a_{j}(s) \leftarrow a_{e}(s) \\
& \text { End if }
\end{aligned}
$$

\section{End for}

\section{End for}

Probabilistically mutate the population $P$ as described in the standard BBO algorithm 


\subsection{Ensemble Multi-objective BBO}

Now the ensemble multi-objective BBO (EMBBO) algorithm is proposed, which is an ensemble of the previously-discussed VEBBO, NSBBO, and NPBBO algorithms. The proposed approach is motivated as follows. According to the no free lunch (NFL) theorem, it is impossible for a single optimization algorithm to outperform all other algorithms on every problem. Each problem has its own characteristics, such as the search space, constraint conditions, objective function landscape, and so on. Different problems require different algorithms, depending on the nature of the problem and available computing resources. In addition, for a given problem, each optimization algorithm may perform differently during different phases of the search process. For example, an algorithm with better exploration may have better search ability during the initial phase of the optimization process, and another algorithm with better exploitation may have better search ability during the later phases of the optimization process. Hence, different optimization algorithms may be suitable during different stages of the search process.

Motivated by these observations, an ensemble of MBBO algorithms composed of VEBBO, NSBBO and NPBBO is implemented in parallel populations in the EMBBO algorithm. The best individuals among the parallel populations are used to yield improved performance using multiple MBBO algorithms. In this paper, three populations are used based on the three previously-discussed MBBO algorithms. More populations can be used if additional MBBO algorithms are used. Each population generates its own offspring. All of the offspring populations are combined to select a fixed number of the best individuals, which updates all of the parent populations. In this way, EMBBO always keeps the individuals that are generated by the more suitable MBBO algorithm, leading to performance that is better than any individual $\mathrm{MBBO}$ algorithm. The flowchart of 
EMBBO is shown in Figure 2, where there are three parallel populations, although less or more could be used, depending on the application. The basic procedure of EMBBO is summarized from Figure 2 as follows.

Step 1. Randomly initialize each parallel parent population $P_{1}, P_{2}$, and $P_{3}$. Use the same population size for each parallel parent population, so each parallel parent population is one-third of the total EMBBO population size.

Step 2. Create offspring populations $O_{1}, O_{2}$, and $O_{3}$, from $P_{1}, P_{2}$, and $P_{3}$ respectively, using VEBBO, NSBBO, and NPBBO respectively.

Step 3. Obtain population $P_{4}$ by selecting a fixed number of the best individuals from the union of $O_{1}, O_{2}$, and $O_{3}$. First, $O_{1}, O_{2}$, and $O_{3}$ are combined into a single population, and then the $\hat{N}$ best individuals are selected from the combined population using non-dominated sorting, where $\hat{N}$ is the population size of $P_{1}$ (which is also the population size of $P_{2}$ and $P_{3}$ ). That is, the best individuals are iteratively added to the new population $P_{4}$ based on levels of non-domination until the desired population size $\hat{N}$ is reached.

Step 4. Update the parallel parent populations $P_{1}, P_{2}$, and $P_{3}$, with $P_{4}$. Each parent population is completely replaced by the new population $P_{4}$. That is, $P_{1}, P_{2}$, and $P_{3}$ are composed of the same individuals after this replacement. Their offspring will be different in the following generation because of the different algorithms in step 2.

Step 5. If the termination criterion is not met, go to step 2; otherwise, terminate. Here the termination criterion is the maximum number of function evaluations, and $P_{4}$ is the output of EMBBO after termination. 




Figure 2 - Flowchart of EMBBO, with three parallel populations $P_{1}, P_{2}$, and $P_{3} . P_{4}$ is the updating population, and $O_{1}, O_{2}$, and $O_{3}$ are offspring populations.

\section{Simulations and Results}

In this section the performance of the proposed EMBBO algorithm is investigated on a set of unconstrained and constrained multi-objective benchmark functions, and on the automated warehouse scheduling problem. Section 4.1 discusses the simulation setup, Section 4.2 presents performance comparisons on the 2009 CEC benchmark functions, and Section 4.3 applies EMBBO to the automated warehouse scheduling problem. 


\subsection{Simulation Setup}

The performance of EMBBO and its constituent algorithms, including VEBBO, NPBBO, and SPBBO, is evaluated on a set of 10 unconstrained functions and 10 constrained functions, which are taken from the CEC 2009 benchmark set. These functions are briefly summarized in Table A in the appendix, where U01-U10 are unconstrained multi-objective benchmark functions, and C01-C10 are constrained multi-objective benchmark functions. U01-U07 and C01-C07 are two-objective problems, and U08-U10 and C08-C10 are three-objective problems. The constrained multi-objective benchmark functions include one or two inequality constraints. The complete definition of each function is available in the literature (Zhang, et al., 2008).

For all algorithms, population size and mutation rate have to be determined. In the literature (Simon, 2008) these parameters have been discussed in detailed. We use a population size of 50 for each parallel constituent algorithm of EMBBO, so the total EMBBO population size is 150 because there are three parallel populations. To obtain fair comparisons, a population size for each individual MBBO (when running by itself apart from EMBBO) is set to 150 . The mutation rate is set to 0.01 per solution decision variable per generation. If mutation occurs, the mutated value of the new independent variable is uniformly distributed in the search space. We evaluate each algorithm 30 times, with a maximum number of function evaluations equal to 300,000 for each simulation.

Hypervolume is used as the performance metric. Suppose that a MOEA has found $M$ points in an

approximate Pareto front $\hat{P}_{f}=\left\{f\left(a_{j}\right)\right\}$ for $j \in[1, M]$, where $f\left(a_{j}\right)$ is a $k$-objective function. The hypervolume can be computed as

$$
Y\left(\hat{P}_{f}\right)=\sum_{j=1}^{M} \prod_{i=1}^{k} f_{i}\left(a_{j}\right)
$$


Given two algorithms that compute two Pareto front approximations to a given MOP, we can use hypervolume to quantify how good the two approximations are relative to each other. For a minimization problem, a smaller hypervolume indicates a better Pareto front approximation.

EMBBO is also compared with the 5 best algorithms from the 2009 CEC competition for unconstrained benchmark functions, including MOEAD, MTS, DMOEADD, LiuLiAlgorithm, and GDE3; and with the 3 best algorithms from the 2009 CEC competition for constrained benchmark functions, including DMOEADD, LiuLiAlgorithm, and MTS. These are the best algorithms from the 13 accepted algorithms in the 2009 CEC competition (Suganthan, 2014).

(a) MOEAD is based on decomposition (Chen, et al., 2009).

(b) MTS is a multiple trajectory search algorithm (Tseng and Chen, 2009).

(c) DMOEADD is an improvement of the dynamic multi-objective evolutionary algorithm and is based on domain decomposition (Liu, et al., 2009).

(d) LiuLiAlgorithm is based on sub-regional search (Liu and Li, 2009).

(e) GDE3 is the third version of a generalized differential evolution algorithm with a diversity maintenance technique (Kukkonen and Lampinen, 2009).

In addition, a Holm multiple comparison test determines statistically significant differences between EMBBO used as the control method, and the other algorithms. The Holm multiple comparison test is a nonparametric statistical test that obtains a probability ( $p$-value) that determines the degree of difference between a control algorithm and a set of alternative algorithms, assuming that the algorithms have statistically significant differences as a whole. To determine whether a set of algorithms shows a statistically significant difference as a whole, Friedman's test is used with a significance level $\alpha=0.1$ to the mean error rankings. If the test rejects the null hypothesis that all 
of the algorithms perform similarly, the best algorithm is considered as the control method and is then compared with the other algorithms according to their rankings. Additional details about the Holm multiple comparison test can be found in the literature (Derrac, et al., 2011).

\subsection{Performance comparisons}

Tables $\mathrm{B}$ and $\mathrm{C}$ in the appendix summarize the performance comparison of EMBBO, VEBBO, NPBBO, SPBBO, and the 2009 CEC competition algorithms on each benchmark function with respect to the hypervolume and normalized hypervolume. Note that hypervolume cannot be blindly used as an indicator of Pareto front quality because if $M$ is larger, then $S$ becomes larger also. But that means a Pareto front with more points would be worse than a Pareto front with fewer points. Normalization takes the number of Pareto front points into account.

For the comparison of EMBBO with its constituent algorithms, Table B shows that for unconstrained benchmark functions, EMBBO performs best on 7 functions (U01, U03, U04, U05, U06, U07 and U09), and NPBBO performs best on 3 functions (U02, U08 and U10). Table C shows that for constrained benchmark functions, EMBBO performs best on 6 functions $(\mathrm{C} 01, \mathrm{C} 03, \mathrm{C} 04$, C06, C07 and C09), NPBBO performs best on 3 functions (C02, C05 and C10), and VEBBO performs best on C08. The proposed EMBBO performs significantly better than the constituent MBBO algorithms for both the unconstrained and constrained multi-objective benchmark functions.

For the comparison of EMBBO with the 2009 CEC competition algorithms, Table B shows that for unconstrained benchmark functions, MOEAD performs best on 5 functions (U01, U02, U04, U09, and U10), MTS performs best on 3 functions (U05, U06, and U08), and EMBBO performs best on 2 functions (U03 and U07). Table $\mathrm{C}$ shows that for constrained benchmark functions, DMOEADD 
performs best on 7 functions (C01, C02, C05, C06, C07, C08 and C10), and EMBBO performs best on 3 functions (C03, C04 and C09). This indicates that for unconstrained benchmark functions, MOEAD is the best, MTS is the second best and EMBBO is the third best. For constrained benchmark functions, DMOEADD is the best and EMBBO is the second best.

The empirical results show that EMBBO is a competitive algorithm for multi-objective benchmark functions. The reasons for its competitive performance are that, first, BBO has distinctive migration behavior compared to other EAs. Second, EMBBO effectively uses ensemble learning. That is, EMBBO uses different $\mathrm{MBBO}$ algorithms in multiple parallel populations to create different offspring populations throughout the search process. By comparing the multiple offspring populations, the best individuals are retained in EMBBO. The average running times of the algorithms are shown in the last row of Tables $\mathrm{B}$ and $\mathrm{C}$ in the appendix. The algorithms are programmed in MATLAB $^{\circledR}$ on a $2.40 \mathrm{GHz}$ Intel Pentium ${ }^{\circledR} 4$ CPU with 4 GB of memory. From the tables, we see that the average running time of EMBBO is much less than the constituent MBBO algorithms. The reason is that EMBBO uses multiple parallel subpopulations of relatively small size, while the constituent $\mathrm{MBBO}$ algorithms use a single population that is three times as large as that of EMBBO. Certain EA operations, such as roulette-wheel selection, require computational effort on the order of $N_{0}^{2}$, where $N_{0}$ is the population size, and so multiple subpopulations are more computationally efficient than a single large population. Multiple subpopulations are also amenable to parallel processing, which can further reduce computational effort.

Table 1 shows the results of the Holm multiple comparison test between EMBBO and its constituent algorithms and the 2009 CEC competition algorithms. For unconstrained functions EMBBO is the third best, and for constrained functions it is the second best. EMBBO is compared 
with its constituent algorithms and the 5 best CEC competition algorithms for unconstrained optimization, and with its constituent algorithms and the 3 best CEC competition algorithms for constrained optimization. Note that Table 1 shows that EMBBO is outperformed by MOEAD and MTS for unconstrained functions and by DMOEADD for constrained functions, as indicated by p-values smaller than 0.1 . Table 1 also shows that EMBBO is statistically significantly better than its constituent algorithms VEBBO, NPBBO, and SPBBO.

Table 1 - Holm multiple comparison test results of EMBBO and its constituent algorithms and 2009 CEC competition algorithms.

\begin{tabular}{ccc|ccc}
\hline \multicolumn{2}{c|}{ Unconstrained functions } & \multicolumn{3}{c}{ Constrained functions } \\
\hline Algorithm & Rank & $p$-value & Algorithm & Rank & $p$-value \\
\hline MOEAD & 1.4 & 0.0076 & DMOEADD & 1.3 & 0.0198 \\
MTS & 2.6 & 0.0227 & EMBBO & 2.7 & - \\
EMBBO & 3.7 & - & LiuLiAlgorithm & 3.7 & 0.0402 \\
DMOEADD & 4.1 & 0.0910 & NPBBO & 3.8 & 0.0421 \\
NPBBO & 4.3 & 0.0613 & MTS & 4.7 & 0.0094 \\
LiuLiAlgorithm & 5.8 & 0.0086 & VEBBO & 4.8 & 0.0086 \\
GDE3 & 6.6 & 0.0021 & NSBBO & 7.0 & 0.0007 \\
VEBBO & 7.2 & 0.0010 & & & \\
NSBBO & 8.8 & 0.0008 & & & \\
\hline
\end{tabular}

\subsection{Application to automated warehouse scheduling}

In this section, the proposed EMBBO is applied to the automated warehouse scheduling problem from Section 2. The width, length and height of each storage unit are $W=0.3 \mathrm{~m}, L=0.5 \mathrm{~m}$ and $H=0.4 \mathrm{~m}$, the distance between adjacent SRs is $D=1.8 \mathrm{~m}$, the number of storage units in each SR is $C=75$, the warehouse throughput capacity is $Q=600$, and the number of products in the warehouse is $q=250$. The $\mathrm{S} / \mathrm{R}$ machine capacity is $N=4$, its horizontal velocity is $v_{x}=1 \mathrm{~m} / \mathrm{s}$, and its vertical velocity is $v_{y}=0.5 \mathrm{~m} / \mathrm{s}$. The required time for each task is the same, which is $120 \mathrm{~s}$. All these parameters are taken from a real-world automated warehouse scheduling problem. We 
consider five implementation schemes. Scheme 1 (number of storage products $m=20$, and number of outgoing products $n=20$ ) is described as follows.

$$
\begin{aligned}
& p_{1}:(12,6,2,50,1), p_{2}:(51,8,1,45,1), p_{3}:(40,3,5,46,1), p_{4}:(37,3,4,113,1), \\
& p_{5}:(14,7,1,40,1), p_{6}:(13,5,2,50,1), p_{7}:(45,5,5,50,1), p_{8}:(18,7,3,33,1), \\
& p_{9}:(7,3,2,35,1), p_{10}:(11,4,3,110,1), p_{11}:(15,2,1,34,1), p_{12}:(36,8,4,40,1), \\
& p_{13}:(8,6,5,47,1), p_{14}:(56,2,3,34,1), p_{15}:(42,8,3,30,1), p_{16}:(23,4,1,50,1), \\
& p_{17}:(4,6,2,50,1), p_{18}:(13,1,1,36,1), p_{19}:(39,6,4,42,1), p_{20}:(45,6,5,55,1) \\
& p_{21}:(50,8,1,67,2), p_{22}:(3,1,2,74,2), p_{23}:(55,4,3,68,2), p_{24}:(6,7,4,85,2), \\
& p_{25}:(17,4,5,74,2), p_{26}:(60,7,3,80,2), p_{27}:(35,6,2,67,2), p_{28}:(15,2,1,70,2), \\
& p_{29}:(57,4,2,80,2), p_{30}:(2,1,4,62,2), p_{31}:(25,8,2,76,2), p_{32}:(5,2,4,64,2), \\
& p_{33}:(17,5,3,76,2), p_{34}:(41,2,5,91,2), p_{35}:(19,3,2,70,2), p_{36}:(20,1,1,82,2), \\
& p_{37}:(42,6,2,88,2), p_{38}:(32,6,3,75,2), p_{39}:(9,7,1,82,2), p_{40}:(58,5,4,69,2)
\end{aligned}
$$

Each storage or retrieval operator is denoted by $(x, y, z, w, u)$, where $x, y$, and $z$ are the Euclidean coordinates of each storage unit (meters), $w$ is the weighting coefficient described in Section 2 which affects the scheduling quality, and $u=1$ indicates a storage product and $u=2$ indicates an outgoing product.

Scheme $2(m=20, n=16)$ includes all the storage products of Scheme 1 but only the first 16 outgoing products of Scheme 1. Scheme $3(m=20, n=12)$ includes all the storage products of Scheme 1 but only the first 12 outgoing products. Scheme $4(m=16, n=20)$ includes the first 16 storage products of Scheme 1 and all of the outgoing products. Scheme $5(m=12, n=20)$ includes the first 12 storage products of Scheme 1 and all of the outgoing products.

The tuning parameters of the MBBO algorithms are the same as those used in the benchmark 
simulations. The optimization results are summarized in Table 2. It is seen from Table 2 that EMBBO performs best for all of the schemes except Scheme 4, for which NPBBO is the best because of its shortest travel distance and its lowest scheduling quality effect. Based on these results, it is concluded that the proposed EMBBO algorithm generally provides better performance than the single-constituent MBBO algorithms for our automated warehouse scheduling problem.

Table 2 - MBBO results for 5 schemes of the automated warehouse scheduling problem. "Distance" denotes the shortest travel distance, which is measured in meters, and "Effect" denotes the lowest scheduling quality effect. The best results in each row are shown in boldface font.

\begin{tabular}{lccccccccc}
\hline \multirow{2}{*}{ Problem } & \multirow{2}{*}{$(m, n)$} & \multicolumn{2}{c}{ VEBBO } & \multicolumn{2}{c}{ NSBBO } & \multicolumn{2}{c}{ NPBBO } & \multicolumn{2}{c}{ EMBBO } \\
& & Distance & Effect & Distance & Effect & Distance & Effect & Distance & Effect \\
\hline Scheme 1 & $(20,20)$ & 129.3 & 2591.3 & 122.2 & 2504.3 & 124.3 & 2555.3 & $\mathbf{1 2 0 . 3}$ & $\mathbf{2 1 7 5 . 6}$ \\
Scheme 2 & $(20,16)$ & 92.5 & 1531.0 & 98.3 & 1562.7 & 89.7 & 1453.6 & $\mathbf{7 7 . 2}$ & $\mathbf{1 3 0 8 . 5}$ \\
Scheme 3 & $(20,12)$ & 71.0 & 826.2 & 63.2 & 857.7 & 62.3 & 798.7 & $\mathbf{5 7 . 1}$ & $\mathbf{7 5 6 . 8}$ \\
Scheme 4 & $(16,20)$ & 109.4 & 1823.8 & 97.6 & 1467.9 & $\mathbf{8 8 . 0}$ & $\mathbf{1 2 9 5 . 5}$ & 88.9 & 1394.0 \\
Scheme 5 & $(12,20)$ & 69.8 & 840.0 & 60.5 & 839.4 & 67.7 & 908.6 & $\mathbf{5 8 . 1}$ & $\mathbf{8 3 6 . 0}$ \\
\hline
\end{tabular}

Table 3 - Scheduling orders as optimized by the proposed EMBBO algorithm. "Route" denotes the route number index, and "Scheduling orders" denotes the scheduling orders that the S/R machine implements each route.

\begin{tabular}{cc}
\hline Route & Scheduling orders \\
\hline 1 & $p_{6} \rightarrow p_{12} \rightarrow p_{3} \rightarrow p_{7} \rightarrow p_{34} \rightarrow p_{23} \rightarrow p_{31} \rightarrow p_{24}$ \\
2 & $p_{16} \rightarrow p_{8} \rightarrow p_{20} \rightarrow p_{2} \rightarrow p_{26} \rightarrow p_{35} \rightarrow p_{28} \rightarrow p_{22}$ \\
3 & $p_{13} \rightarrow p_{10} \rightarrow p_{11} \rightarrow p_{15} \rightarrow p_{29} \rightarrow p_{40} \rightarrow p_{36} \rightarrow p_{30}$ \\
4 & $p_{9} \rightarrow p_{1} \rightarrow p_{18} \rightarrow p_{4} \rightarrow p_{37} \rightarrow p_{21} \rightarrow p_{25} \rightarrow p_{39}$ \\
5 & $p_{17} \rightarrow p_{5} \rightarrow p_{19} \rightarrow p_{14} \rightarrow p_{27} \rightarrow p_{38} \rightarrow p_{33} \rightarrow p_{32}$ \\
\hline
\end{tabular}

A sample EMBBO scheduling route output is shown in Table 3 for Scheme 1. It is seen that the automated warehouse scheduling problem is divided into 5 routes, and each route includes 8 storage units, where the first 4 storage units are used to store products, and the last 4 storage units are used to retrieve products. 
Next we compare EMBBO with the 3 best constrained optimization algorithms from the CEC 2009 competition, which include DMOEADD, LiuLiAlgorithm, and MTS. The optimization results are summarized in Table 4. It is seen that DMOEADD performs best on 3 cases (Scheme 1, Scheme 3, and Scheme 5), and EMBBO performs best on the other 2 cases (Scheme 2 and Scheme 4). In the 3 schemes for which DMOEADD performed best, EMBBO performed second best. Although EMBBO is not the best algorithm, it is a consistently competitive algorithm for the automated warehouse scheduling problem.

Table 4 - Comparisons between EMBBO, DMOEADD, LiuLiAlgorithm, and MTS for the automated warehouse scheduling problem. "Distance" denotes the shortest travel distance, which is measured in meters, and "Effect" denotes the lowest scheduling quality effect. The best results in each row are shown in boldface font.

\begin{tabular}{lccccccccc}
\hline \multirow{2}{*}{ Problem } & \multirow{2}{*}{$(m, n)$} & \multicolumn{2}{c}{ DMOEADD } & \multicolumn{2}{c}{ LiuLiAlgorithm } & \multicolumn{2}{c}{ MTS } & \multicolumn{2}{c}{ EMBBO } \\
& & Distance & Effect & Distance & Effect & Distance & Effect & Distance & Effect \\
\hline Scheme 1 & $(20,20)$ & $\mathbf{1 1 4 . 4}$ & $\mathbf{2 0 4 7 . 5}$ & 130.1 & 2314.6 & 136.7 & 2411.5 & 120.3 & 2175.6 \\
Scheme 2 & $(20,16)$ & 84.5 & 1612.2 & 89.4 & 1639.1 & 92.1 & 1694.3 & $\mathbf{7 7 . 2}$ & $\mathbf{1 3 0 8 . 5}$ \\
Scheme 3 & $(20,12)$ & $\mathbf{5 0 . 3}$ & $\mathbf{7 2 1 . 5}$ & 62.4 & 779.2 & 62.9 & 801.6 & 57.1 & 756.8 \\
Scheme 4 & $(16,20)$ & 91.5 & 1486.1 & 93.4 & 1507.6 & 99.1 & 1611.2 & $\mathbf{8 8 . 9}$ & $\mathbf{1 3 9 4 . 0}$ \\
Scheme 5 & $(12,20)$ & $\mathbf{5 2 . 4}$ & $\mathbf{8 1 1 . 9}$ & 61.0 & 853.7 & 62.7 & 892.3 & 58.1 & 836.0 \\
\hline
\end{tabular}

\section{Conclusions}

In this paper an ensemble multi-objective biogeography-based optimization algorithm called EMBBO was proposed to solve general multi-objective optimization problems, both constrained and unconstrained. In addition, an automated warehouse scheduling model was built based on common industrial warehouse characteristics, and the scheduling problem was formulated as a constrained multi-objective optimization problem.

The performance of EMBBO was investigated on a set of CEC 2009 multi-objective benchmark functions. The numerical simulations showed that EMBBO is better than single-constituent MBBO 
algorithms for the most of the benchmark functions. In particular, EMBBO is better than its constituent algorithm on 7 of 10 unconstrained benchmarks, and 6 of 10 constrained benchmarks. Furthermore, EMBBO is competitive with the best CEC 2009 algorithms for multi-objective optimization. In particular, EMBBO is better than the best CEC 2009 algorithms on 5 of 10 unconstrained benchmarks, and 3 of 10 constrained benchmarks.

Finally, EMBBO was applied to the automated warehouse scheduling problem and the results again showed that EMBBO is a competitive optimization method. In particular, EMBBO was better than its constituent algorithms on 4 of 5 warehouse scheduling problems, and it was better than the best CEC 2009 algorithms on 2 of 5 warehouse scheduling problems.

Based on the optimization tests in this paper, EMBBO can effectively improve the performance of $\mathrm{MBBO}$ on multi-objective optimization problems, including real-world warehouse scheduling. The fundamental contributions of this paper are twofold. First, multiple MBBO algorithms are combined into a single algorithm, leveraging the best features of multiple algorithms. The framework presented here could be extended for other types of optimization algorithms also. Second, a real-world warehouse scheduling problem was formulated in a way that is amenable to multi-objective evolutionary algorithms.

\section{Acknowledgments}

This material is based upon work supported by the National Science Foundation under Grant No.

1344954, the National Natural Science Foundation of China under Grant No. 61305078, 61074032, 61179041, and the Shaoxing City Public Technology Applied Research Project under Grant No. 2013 B70004. 


\section{Appendix}

The abbreviations, notations, and symbols used in this paper are summarized as follows.

BBO: Biogeography-based optimization.

EMBBO: Ensemble multi-objective biogeography-based optimization.

MBBO: Multi-objective biogeography-based optimization.

VEBBO: Vector evaluated biogeography-based optimization.

NSBBO: Non-dominated sorting biogeography-based optimization.

NPBBO: Niched Pareto biogeography-based optimization.

GA: Genetic algorithm.

VEGA: Vector evaluated genetic algorithm.

NSGA: Non-dominated sorting genetic algorithm.

NPGA: Niched Pareto genetic algorithm.

ES: Evolution strategies.

EP: Evolutionary programming.

HS: Harmony search.

DE: Differential evolution.

MOEA: Multi-objective evolutionary algorithm.

MOP: Multi-objective optimization problem.

CEC: Congress on Evolutionary Computation.

AS/RS: Automated storage and retrieval systems

S/R: Storage and retrieval.

SR: Storage rack.

I/O: Input/output.

$\operatorname{rand}(\omega, \sigma)$ : Uniformly distributed random number between $\omega$ and $\sigma$.

$\lceil\varphi\rceil:$ Smallest integer greater than or equal to $\varphi$.

$B$ : Population of non-dominated individuals.

$C:$ Number of storage units in each SR.

$D$ : Distance between adjacent SRs.

$H$ : Height of each storage unit.

$N$ : Number of products S/R machine bears. 
$N_{0}$ : Population size.

$O$ : Offspring population.

$P$ : Population of candidate solutions.

$Q:$ Warehouse throughput capacity.

$S$ : Subset of the population.

$T$ : Temporary population.

$T_{r}$ : Specified scheduling time.

$W:$ Width of each storage unit.

$Y$ : Hypervolume.

$L_{s}$ : Lower search bounds of the sth independent variable.

$U_{s}$ : Upper search bounds of the sth independent variable.

$a$ : Candidate solution.

$a_{0}$ : Non-dominated individual.

$b_{i}$ : Number of individuals that dominate $a_{i}$.

$c:$ Non-domination level.

$c_{i}$ : Crowding distance of $a_{i}$.

$d_{i j}$ : Travel distance of S/R machine.

$f$ : Candidate solution fitness.

$k:$ Number of objectives.

$l, g, e:$ Flag sign.

$m:$ Number of storage products.

$n$ : Number of outgoing products.

$n_{0}$ : Optimization problem dimension.

$p$ : Storage unit.

$q$ : Number of products in the warehouse

$r$ : Route S/R machine goes through.

$s$ : Decision variable.

$t_{i j}$ : Time of S/R machine operation.

$v_{x}$ : Horizontal velocity.

$v_{y}$ : Vertical velocity.

$w_{r}$ : Weight coefficient. 
$x, y, z$ : Euclidean coordinates.

$\gamma:$ Rank of a solution with respect to a given objective.

\section{$\lambda$ : Immigration rate.}

$\mu$ : Emigration rate.

$\theta:$ Mutation rate.

Table A- CEC 2009 multi-objective benchmark functions, where $n_{0}$ denotes the number of dimensions in each objective. More detailed about these functions can be found in (Zhang, et al., 2008).

\begin{tabular}{|c|c|c|c|}
\hline & Function name & Num. of objectives & Search space \\
\hline \multirow{10}{*}{$\begin{array}{l}\text { Unconstrained } \\
\text { multi-objective } \\
\text { benchmark } \\
\text { functions }\end{array}$} & U01: unconstrained problem 1 & 2 & {$[0,1] \times[-1,1]^{n_{0}-1}, \quad n_{0}=30$} \\
\hline & U02: unconstrained problem 2 & 2 & {$[0,1] \times[-1,1]^{n_{0}-1}, \quad n_{0}=30$} \\
\hline & U03: unconstrained problem 3 & 2 & {$[0,1]^{n_{0}}, \quad n_{0}=30$} \\
\hline & U04: unconstrained problem 4 & 2 & {$[0,1] \times[-2,2]^{n_{0}-1}, \quad n_{0}=30$} \\
\hline & U05: unconstrained problem 5 & 2 & {$[0,1] \times[-1,1]^{n_{0}-1}, \quad n_{0}=30$} \\
\hline & U06: unconstrained problem 6 & 2 & {$[0,1] \times[-1,1]^{n_{0}-1}, \quad n_{0}=30$} \\
\hline & U07: unconstrained problem 7 & 2 & {$[0,1] \times[-1,1]^{n_{0}-1}, \quad n_{0}=30$} \\
\hline & U08: unconstrained problem 8 & 3 & {$[0,1]^{2} \times[-2,2]^{n_{0}-2}, \quad n_{0}=30$} \\
\hline & U09: unconstrained problem 9 & 3 & {$[0,1]^{2} \times[-2,2]^{n_{0}-2}, \quad n_{0}=30$} \\
\hline & U10: unconstrained problem 10 & 3 & {$[0,1]^{2} \times[-2,2]^{n_{0}-2}, \quad n_{0}=30$} \\
\hline \multirow{10}{*}{$\begin{array}{c}\text { Constrained } \\
\text { multi-objective } \\
\text { benchmark } \\
\text { functions }\end{array}$} & C01: Constrained problem 1 & 2 & {$[0,1]^{n_{0}}, \quad n_{0}=10$} \\
\hline & C02: Constrained problem 2 & 2 & {$[0,1] \times[-1,1]^{n_{0}-1}, \quad n_{0}=10$} \\
\hline & C03: Constrained problem 3 & 2 & {$[0,1] \times[-2,2]^{n_{0}-1}, \quad n_{0}=10$} \\
\hline & C04: Constrained problem 4 & 2 & {$[0,1] \times[-2,2]^{n_{0}-1}, \quad n_{0}=10$} \\
\hline & C05: Constrained problem 5 & 2 & {$[0,1] \times[-2,2]^{n_{0}-1}, \quad n_{0}=10$} \\
\hline & C06: Constrained problem 6 & 2 & {$[0,1] \times[-2,2]^{n_{0}-1}, \quad n_{0}=10$} \\
\hline & C07: Constrained problem 7 & 2 & {$[0,1] \times[-2,2]^{n_{0}-1}, \quad n_{0}=10$} \\
\hline & C08: Constrained problem 8 & 3 & {$[0,1]^{2} \times[-4,4]^{n_{0}-2}, \quad n_{0}=10$} \\
\hline & C09: Constrained problem 9 & 3 & {$[0,1]^{2} \times[-2,2]^{n_{0}-2}, \quad n_{0}=10$} \\
\hline & C10: Constrained problem 10 & 3 & {$[0,1]^{2} \times[-2,2]^{n_{0}-2}, \quad n_{0}=10$} \\
\hline
\end{tabular}


Table B - MBBO and CEC competition algorithm results for 10 unconstrained multi-objective benchmark functions. The table shows the relative hypervolume and normalized relative hypervolume. The best results with respect to MBBO in each row are shown in boldface font. The best results among all algorithms are underlined.

\begin{tabular}{|c|c|c|c|c|c|c|c|c|c|}
\hline \multirow{2}{*}{ Fun. } & \multicolumn{4}{|c|}{ MBBO } & \multicolumn{5}{|c|}{ CEC competition algorithms } \\
\hline & VEBBO & NSBBO & NPBBO & EMBBO & MOEAD & MTS & DMOEADD & LiuLiAlgorithm & GDE3 \\
\hline U01 & $(58.59,0.133)$ & $(73.61,0.167)$ & $(51.56,0.117)$ & $(40.48,0.092)$ & $(\underline{37.47,0.085)}$ & $(39.45,0.089)$ & $(44.36,0.101)$ & $(47.59,0.108)$ & $(47.35,0.107)$ \\
\hline U02 & $(24.39,0.123)$ & $(30.58,0.155)$ & $(17.37,0.088)$ & $(20.85,0.106)$ & $(\underline{17.16,0.087})$ & $(17.85,0.091)$ & $(22.44,0.113)$ & $(22.82,0.115)$ & $(23.78,0.120)$ \\
\hline U03 & $(288.9,0.122)$ & $(398.4,0.168)$ & $(232.9,0.098)$ & $(\underline{202.1,0.085})$ & $(203.1,0.086)$ & $(230.9,0.097)$ & $(247.3,0.104)$ & $(280.2,0.118)$ & $(294.6,0.124)$ \\
\hline U04 & $(8.549,0.109)$ & $(11.45,0.146)$ & $(8.373,0.107)$ & $(7.900,0.100)$ & $(\underline{7.413,0.095})$ & $(8.070,0.103)$ & $(9.334,0.119)$ & $(8.940,0.114)$ & $(8.311,0.106)$ \\
\hline U05 & $(286.7,0.192)$ & $(282.9,0.190)$ & $(130.8,0.088)$ & $(117.1,0.079)$ & $(110.7,0.074)$ & $(\underline{102.8,0.069})$ & $(135.6,0.091)$ & $(155.2,0.104)$ & $(167.1,0.112)$ \\
\hline U06 & $(697.5,0.145)$ & $(795.4,0.169)$ & $(485.4,0.103)$ & $(477.2,0.101)$ & $(425.5,0.090)$ & $(\underline{416.3,0.088})$ & $(446.9,0.095)$ & $(438.5,0.093)$ & $(532.2,0.113)$ \\
\hline U07 & $(55.72,0.124)$ & $(57.96,0.129)$ & $(40.51,0.090)$ & $(\underline{40.40,0.089})$ & $(42.03,0.093)$ & $(45.91,0.102)$ & $(51.34,0.114)$ & $(60.86,0.135)$ & $(55.34,0.123)$ \\
\hline U08 & $(550.4,0.111)$ & $(763.2,0.153)$ & $(428.8,0.086)$ & $(633.5,0.127)$ & $(456.7,0.092)$ & $(\underline{422.5,0.085})$ & $(559.3,0.112)$ & $(572.1,0.115)$ & $(578.9,0.116)$ \\
\hline U09 & $(2003.4,0.124)$ & $(2447.6,0.152)$ & $(1870.1,0.116)$ & $(1476.8,0.092)$ & $(\underline{1305.9,0.081)}$ & $(1403.7,0.087)$ & $(1866.5,0.116)$ & $(1749.3,0.109)$ & $(1988.5,0.123)$ \\
\hline U10 & $(2526.9,0.123)$ & $(3505.4,0.170)$ & $(1811.7,0.088)$ & $(2113.5,0.103)$ & $(\underline{1755.6,0.085})$ & $(2007.5,0.098)$ & $(2016.8,0.098)$ & $(2044.1,0.099)$ & $(2789.6,0.136)$ \\
\hline Time & 347.16 & 645.42 & 304.71 & 237.59 & 221.72 & 277.50 & 242.31 & 292.13 & 198.75 \\
\hline
\end{tabular}

Table C - MBBO and CEC competition algorithm results for 10 constrained multi-objective benchmark functions. The table shows the relative hypervolume and normalized relative hypervolume. The best results with respect to $\mathrm{MBBO}$ in each row are shown in boldface font. The best results among all algorithms are underlined. Average CPU

\begin{tabular}{|c|c|c|c|c|c|c|c|}
\hline \multirow{2}{*}{ Fun. } & \multicolumn{4}{|c|}{ MBBO } & \multicolumn{3}{|c|}{ CEC competition algorithms } \\
\hline & VEBBO & NSBBO & NPBBO & EMBBO & MTS & DMOEADD & LiuLiAlgorithm \\
\hline $\mathrm{C} 01$ & $(6.240,0.185)$ & $(6.545,0.193)$ & $(4.254,0.126)$ & $(3.853,0.114)$ & $(5.256,0.156)$ & $(\underline{3.471,0.103})$ & $(4.178,0.124)$ \\
\hline $\mathrm{C} 02$ & $(15.53,0.171)$ & $(20.41,0.225)$ & $(8.040,0.088)$ & $(9.844,0.108)$ & $(17.20,0.190)$ & $(\underline{7.233,0.081)}$ & $(7.639,0.085)$ \\
\hline $\mathrm{C} 03$ & $(723.6,0.181)$ & $(730.5,0.183)$ & $(529.6,0.133)$ & $(\underline{458.1,0.115})$ & $(607.8,0.152)$ & $(508.6,0.122)$ & $(519.5,0.130)$ \\
\hline $\mathrm{C} 04$ & $(9.890,0.141)$ & $(15.30,0.219)$ & $(7.199,0.103)$ & $(\underline{5.912,0.084})$ & $(13.17,0.188)$ & $(5.989,0.085)$ & $(6.361,0.099)$ \\
\hline $\mathrm{C} 05$ & $(39.29,0.123)$ & $(73.61,0.232)$ & $(30.89,0.097)$ & $(34.56,0.109)$ & $(62.90,0.198)$ & $(\underline{30.80,0.096})$ & $(40.13,0.126)$ \\
\hline $\mathrm{C} 06$ & $(0.280,0.155)$ & $(0.561,0.311)$ & $(0.238,0.131)$ & $(0.191,0.105)$ & $(0.185,0.102)$ & $(\underline{0.162,0.089})$ & $(0.187,0.103)$ \\
\hline $\mathrm{C} 07$ & $(54.11,0.105)$ & $(152.1,0.296)$ & $(50.28,0.097)$ & $(36.62,0.071)$ & $(110.4,0.215)$ & $(\underline{31.30,0.061)}$ & $(48.65,0.090)$ \\
\hline $\mathrm{C} 08$ & $(111.7,0.109)$ & $(186.1,0.181)$ & $(121.6,0.118)$ & $(159.3,0.155)$ & $(138.7,0.135)$ & $(\underline{109.5,0.105})$ & $(177.3,0.173)$ \\
\hline $\mathrm{C} 09$ & $(137.0,0.152)$ & $(176.9,0.197)$ & $(155.2,0.173)$ & $(\underline{\mathbf{1 0 5 . 0}, 0.117})$ & $(112.4,0.125)$ & $(109.41,0.120)$ & $(120.8,0.134)$ \\
\hline $\mathrm{C} 10$ & $(1439.5,0.113)$ & $(2631.5,0.207)$ & $(1334.8,0.105)$ & $(1508.1,0.118)$ & $(2170.2,0.171)$ & $(\underline{1157.6,0.091)}$ & $(2438.4,0.192)$ \\
\hline Time & 322.30 & 513.75 & 284.24 & 214.42 & 256.87 & 227.63 & 270.35 \\
\hline
\end{tabular}




\section{References}

Berg, J. P., 1999. A literrauture survey on planning and control of warehousing systems, IIE Transactions, 31, $751-762$.

Chan, F. T. S., Kumar, V., 2009. Hybrid TSSA algorithm-based approach to solve warehouse-scheduling problems, International Journal of Production Research, 47 (4), 919 940.

Chen, C., Chen, Y., Zhang, Q., 2009. Enhancing MOEA/D with Guided Mutation and Priority Update for Multi-objective Optimization, In: Proceedings of the IEEE Congress on Evolutionary Computation, Trondheim, 2009, pp. 209 - 216.

Choi, T. M., Yeung, W. K., Cheng, T. C. E., 2013. Scheduling and co-ordination of multi-suppliers single-warehouse-operator single-manufacturer supply chains with variable production rates and storage costs, Ingernational Journal of Production Research, 51(9), 2593 - 2601.

Chutima, P., Wong, N., 2014. A Pareto biogeography-based optimisation for multi-objective two-sided assembly line sequencing problems with a learning effect, Computers and Industrial Engineering, 69 (1), $89-104$.

Coello, C. A. C., Pulido, G. T., Lechuga, M. S., 2004. Handling multiple objectives with particle swarm optimization, IEEE Transactions on Evolutionary Computation, 8(3), 256 - 279.

Costa e Silva, E. M. A., Coelho, L. D. S., Lebensztajn, L., 2012. Multiobjective biogeography-based optimization based on predator-prey approach, IEEE Trans. on Magnetics, $48(2), 951-954$.

Deb, K., Pratap, A., Agarwal, S., Meyarivan, T., 2002. A fast and elitist multi-objective genetic algorithm: NSGA-II, IEEE Trans. on Evolutionary computation, 6(2), 182 - 197. 
Derrac, J., Garcia, S., Molina, D., Herrera, F., 2011. A practical tutorial on the use of nonparametric statistical tests as a methodology for comparing evolutionary and swarm intelligence algorithms, Swarm and Evolutionary Computation, 1 (1), 3 - 18.

Gagliardi, J. P., Renaud, J., Ruiz, A., 2012. Models for automated storage and retrieval systems: a literature review, Interantional Journal of Production Research, 50 (24), 7110 -7125.

Horn, J., Nafpliotis, N., Goldberg, D. E., 1994. A niched Pareto genetic algorithm for multi-objective optimization. In: Proceeding of IEEE World Congress on Computational Intelligence, 1994, pp. $82-87$.

Jamuna, K., Swarup, K. S., 2012. Multi-objective biogeography based optimization for optimal PMU placement, Applied Soft Computing, 12 (5), 1503 - 1510.

Kukkonen, S., Lampinen, J., 2009. Performance Assessment of Generalized Differential Evolution3 (GDE3) with a Given Set of Constrained Multi-Objective Optimization Problems. In: Proceedings of the IEEE Congress on Evolutionary Computation, Trondheim, 2009, pp. 3593 $-3600$.

Lerher, T., Edl, M., Rosi, B., 2013. Energy efficiency model for the mini-load automated storage and retrieval systems, International Journal of Advanced Manufacturing Technology, 70 (1), $97-115$.

Lerher, T., Ekren, B.Y., Dukic, G., Rosi, B., 2015a. Travel time model for shuttle-based storage and retrieval systems, International Journal of Advanced Manufacturing Technology, 40 (3), 101 $-121$.

Lerher, T. Ekren, B. Y., Sari, Z., Rosi, B., 2015b. Simulation analysis of shuttle based storage and retrieval systems, International Journal of Simulation Modelling, 14 (1), 11 -23. 
Lerher, T., Potrc, I., Sraml, M., Tollazzi, T., 2010. Travel time models for automated warehouses with aisle transferring storage and retrieval machine, European Journal of Operational Research, 205 (3), $571-583$.

Lerher, T., Sraml, M., Potrc, I., Tollazzi, T., 2010. Travel time models for double-deep automated storage and retrieval systems, International Journal of Production Research, 48 (11), 3151 -3172 .

Lerher, T., Sraml, M., Potrc, I., 2011. Simulation analysis of mini-load multi-shuttle automated storage and retrieval systems, International Journal of Advanced Manufacturing Technology, $54(1), 337-348$.

Liu, M., Zou, X., Chen, Y., 2009. Performance Assessment of DMOEA-DD with CEC 2009 MOEA Competition Test Instances. In: Proceedings of the IEEE Congress on Evolutionary Computation, Trondheim, 2009, pp. 2913 - 2918.

Liu, H., Li, X., 2009. The multiobjective evolutionary algorithm based on determined weights and sub-regional search, In: Proceedings of the IEEE Congress on Evolutionary Computation, Trondheim, 2009, pp. $1928-1934$.

Mallipeddi, R., Suganthan, P. N., 2010a. Ensemble strategies with adaptive evolutionary programming, Information Sciences, 180 (9), 1571-1581.

Mallipeddi, R., Suganthan, P. N., 2010b. Ensemble of constraint handling techniques, IEEE Trans. on Evolutionary Computation, 14 (4), 561 - 579.

Mallipeddi, R., Suganthan, P. N., Pan, Q. K., Tasgetiren, M. F., 2011. Differential evolution algorithm with ensemble of parameters and mutation strategies, Applied Soft Computing, 11 (2),1679-1696. 
Ma H., Simon, D., 2011. Blended biogeography-based optimization for constrained optimization, Engineering Applications of Artificial Intelligence, 24 (3), 517 - 525.

Ma, H., Ruan, X., Pan, Z., 2012. Handling multiple objectives with biogeography-based optimization, International Journal of Automation and Computing, 9 (1), 30 - 36.

Pan, Q. K., Suganthan, P. N., Tasgetiren, M. F., 2009. A harmony search algorithm with ensemble of parameter sets, IEEE Congress on Evolutionary Computation, Norway, 2009, pp. 1815 1820.

Roodbergen, K. J., Vis, I. F. A., 2009. A survey of literature on automated storage and retrieval systems, European Journal of Operational Research, 194, 343 - 362.

Srinivas, N., Deb, K., 1995. Multi-objective optimization using non-dominated sorting in genetic algorithms. Evolutionary Computation, 2 (3), 221 - 248.

Schaffer, C., 1985. Multiple objective optimization with vector evaluated genetic algorithms, International Conference on Genetic Algorithms and Their Application, Pittsburgh, Pennsylvania, 1985, pp. $93-100$.

Suganthan, P. N., http://www3.ntu.edu.sg/home/EPNSugan/.

Simon, D., 2008. Biogeography-based optimization. IEEE Trans. on Evolutionary Computation, 12 (6), $702-713$.

Simon, D., 2013. Evolutionary Optimization Algorithms, Hoboken, NJ: John Wiley \& Sons.

Tasgetiren, M. F., Suganthan, P. N., Pan, Q-K, Mallipeddi, R., Sarman, S., 2010a. An ensemble of differential evolution algorithms for constrained function optimization, In: Proceedings of the IEEE Congress on Evolutionary Computation, Barcelona, Spain, 2010, pp. 967 - 975. 
Tasgetiren, M. F., Suganthan, P. N., Pan, Q. K., 2010b. An ensemble of discrete differential evolution algorithms for solving the generalized traveling salesman problem, Applied Mathematics and Computation, 215 (9), 3356 - 3368.

Tseng, L., Chen, C., 2009. Multiple Trajectory Search for Unconstrained/Constrained Multi-Objective Optimization. In: Proceedings of the IEEE Congress on Evolutionary Computation, Trondheim, 2009, pp. 1951 - 1958.

Wang, L., Fang, C., 2011. An effective shuffled frog-leaping algorithm for multi-mode resource-constrained project scheduling problem, Information Sciences, 181(20), $4804-4822$.

Yang, W., Deng, L., Niu, Q., Fei, M., 2013. Improved shuffled frog leaping algorithm for solving multi-aisle automated warehouse scheduling optimization, Communications in Computer and Information Science, 402, 82 - 92.

Yeung, W. K., Choi, T. M., Cheng, T. C. E., 2010. Optimal scheduling in a single-supplier single-manufacturer supply chain with common due windows, IEEE Transactions on Automatic Control, 55, 2767 -2777.

Yeung, W. K., Choi, T. M., Cheng, T. C. E., 2011, Supply chain scheduling and coordination with dual delivery modes and inventory storage cost, International Journal of Production Economics, 132, $223-229$.

Yu, E. L., Suganthan, P. N., 2009. Evolutionary programming with ensemble of external memories for dynamic optimization, In: Proceedings of the IEEE Congress on Evolutionary Computation, Norway, 2009, pp. $431-438$. 
Zhao, S. Z., Suganthan, P. N., 2010. Multi-objective evolutionary algorithm with ensemble of external archives, International Journal of Innovative Computing, Information and Control, 6 (1), $1713-1726$.

Zhang, Q., Zhou, A., Zhao, S. Z., Suganthan, P. N., Liu, W., Tiwari, S., 2008. Multi-objective Optimization Test Instances for the CEC 2009 Special Session and Competition, Technical Report. 